\title{
Preliminary investigation of fluid flow in meso-scale rectangular tube
}

\author{
Liang Hu, Qi Kang, Jia-Feng Zhang, Li Duan, Wen-Rui Hu \\ National Microgravity Laboratory/CAS; \\ Institute of Mechanics, Chinese Academy of Sciences, \\ Beijing 100080, China
}

\begin{abstract}
The fluid flow associated with micro and meso scale devices is currently of interest. Experiments were performed to study the fluid flow in meso-scale channels. A straight flow tube was fabricated with $1.0 \times 4.0 \mathrm{~mm}^{2}$ in rectangular cross section and $200 \mathrm{~mm}$ in length, which was made of quartz for flow visualization and PIV measurements. Reynolds numbers were ranged from 311 to over 3105 . The corresponding pressure drop was from $0.65 \mathrm{KPa}$ to over $16.58 \mathrm{KPa}$ between the inlet and outlet of the tube. The micro PIV was developed to measure the velocity distribution in the tube. A set of microscope object lens was mounted ahead of CCD camera to obtain optimized optical magnification on the CCD chip. The velocity distributions near the outlet of the tube were measured to obtain full-developed flow. A CW laser beam was focused directly on the test section by a cylinder lens to form a small light sheet. Thus, high power density of light was formed on the view region. It is very important to the experiment while the velocity of the flow reaches to a few meters per second within millimeter scale. In this case, it is necessary to reduce exposure time to microseconds for PIV measurements. In the present paper, the experimental results are compared with the classical theories.
\end{abstract}

Keyword: micro PIV, mesoscale channel, turbulence, laminar flow

\section{Introduction}

In the last two decades, the art of 'Lab-on-a-Chip' extended explosively. The motivation is, in Gad-el-Hak's words, humans have always striven to explore, build, and control the extremes of length and time scales 0 The problem was prompted by the introduction of the concept of microchannel heat sinks for electronic cooling by Tuckerman and Pease 0 . Nowadays, in addition to the ultra compact heat exchanger, the applications of micro fluid devices had expanded to vast fields. For instance, the biomedical industry is one of the major industries to benefit from the advancement of micro-fluidics. Portable micro-total analysis systems (mTAS) and lab on chips are more close to realization due to current microfluidic techniques. The applications of fluidic MEMS technology also outstretch to observation of chemical reactions, inkjet print heads, and transducers for microfluidic sensor or actuator such as particle sensing, measurement of density, flow, viscosity etc.

Currently, a number of publications indicated that flow features on the microscale are different from the macroscale and that the classical Navier-Stokes equations are limited to explain the phenomena [1,3-8]. However, some studies reported that the measured friction factor of full-developed flow in micro tube was accurately predicted using traditional macroscale theory for some certain regimes [2,9-17]. Perhaps, the reason lay in the different experimental conditions and research emphasis, such as roughness of tube surface, electrical charge of the interfaces, entrance effect of the tube, measurement accuracy etc. Hence, it is essential to study the fluid flow in the microscale to improve the design and fabrication of microfluidic devices.

Santiago et al has developed a micro PIV by combining epi-fluorescent microscope with the PIV technique [18]. Meinhart et al presented measurement of a pressure driven flow field in a $30 \mu \mathrm{m}$ 
$\times 300 \mu \mathrm{m} \times 25 \mathrm{~mm}$ rectangular micro channel by using the $\mu$ PIV [16]. Molho et al studied the velocity profile of the electrokinetically driven flow by using caged fluorescence imaging technique [17]. Also the Optical Doppler Tomographic image used to obtain the point velocity by Chen et al [19]. The bulk flow experiments were conducted to obtain pressure drop and flow rate with different sizes geometries and test fluid $[3,12]$.

In this study, we investigated the flow patterns, velocity fields, pressure drop and flow rate in a $1 \mathrm{~mm} \times 4 \mathrm{~mm} \times 200 \mathrm{~mm}$ rectangular quartz channel.

\section{Experimental setup}

\subsection{Flow loop}

The flow loop is shown in Fig. 1. A gear pump was employed to pump deionized water was pumped from liquid reservoir. The water flow regulated by the by-pass loop and the valves. The differential pressure transducer was installed at the inlet and outlet to measure the pressure drop of the test channel. The dimension of the tube is $1 \mathrm{~mm} \times 4 \mathrm{~mm} \times 200 \mathrm{~mm}$. The length of the test tube is long enough to eliminate entrance effect. The liquid left the test section and then led to the liquid collection beaker. By weighing the collection container over a certain period of time, the mass flow rate could be obtained accurately.

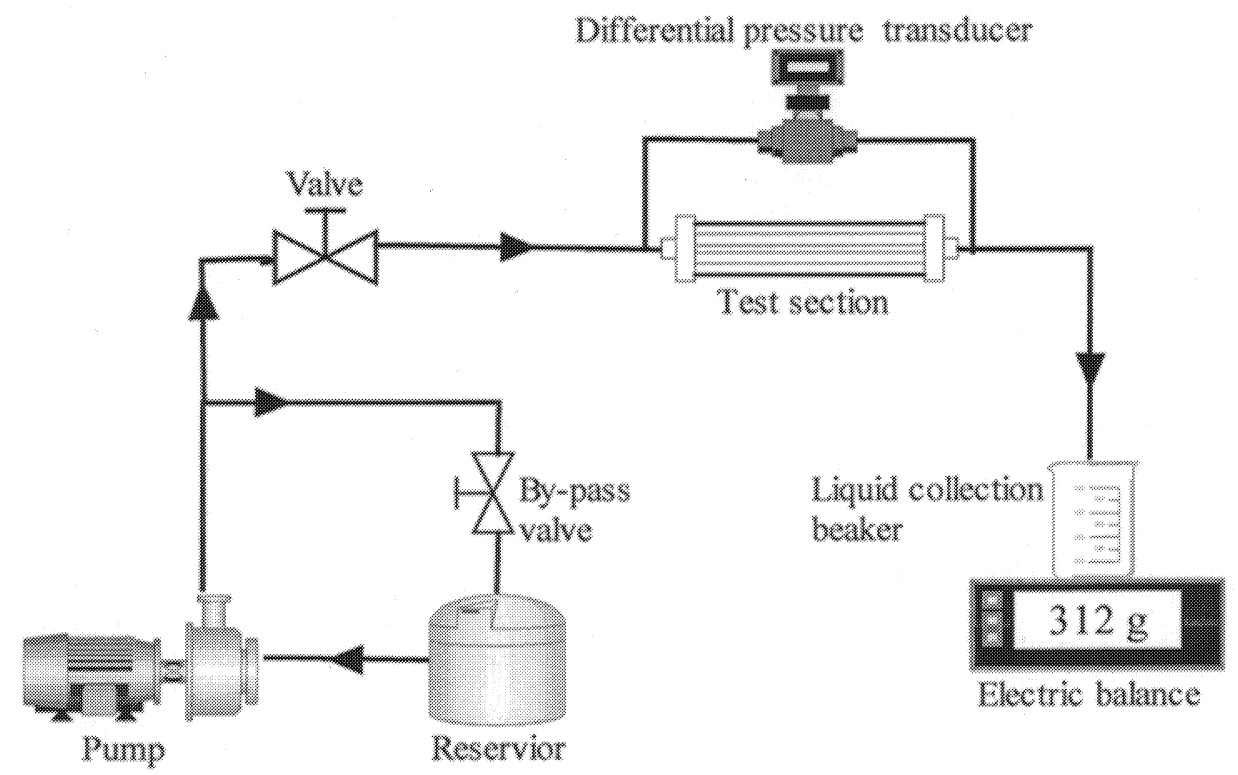

Fig. 1. Schematic of flow loop

\subsection{Optical diagnostic setup}

Fig. 2 shows the configuration of optical diagnostic setup. Dantec Dynamics' silver coated hollow glass spheres were seeded in the working liquid to trace the flow. The mean diameter is about $10 \mu \mathrm{m}$ which can partly meet the requirements suggested by Santiago et al [18], with (1) suitable image size, (2) low Brownian motion effect, without (1) disrupting the flow field, (2) blocking the tube. The flow was illuminated by a CW Ar-ion laser (Stabilite 2017, Spectra Physics Inc.). The maximum output power is about $2 \mathrm{~W}$, which is not enough for PIV measurement in high Reynolds number. The light was modulated to laser pulses by passing a electro-optical shatter (Flowmap 80X41, Dantec Dynamics). The laser beam was focused on the test region by a cylinder lens to obtain high density. The width of light sheet was at the same order of the investigated region. Considering the spatial resolution of the CCD camera, it is volume illumination rather than a thin light sheet. The tube was 
fabricated by quartz which is transparent for visualization of the flow. The roughness of the tube is about $1 \mu \mathrm{m} \sim 10 \mu \mathrm{m}$. The images of tracing particles were captured by a CCD camera with a $768 \times 484$ pixels array and 8 bit resolution (80C42 Double Image 700, Dantec Dynamics), after they were magnified by a set of microscope lens. The synchronization of the shutter camera and image grabber was controlled by a Dantec Dynamic' Flowmap PIV2000 processor.

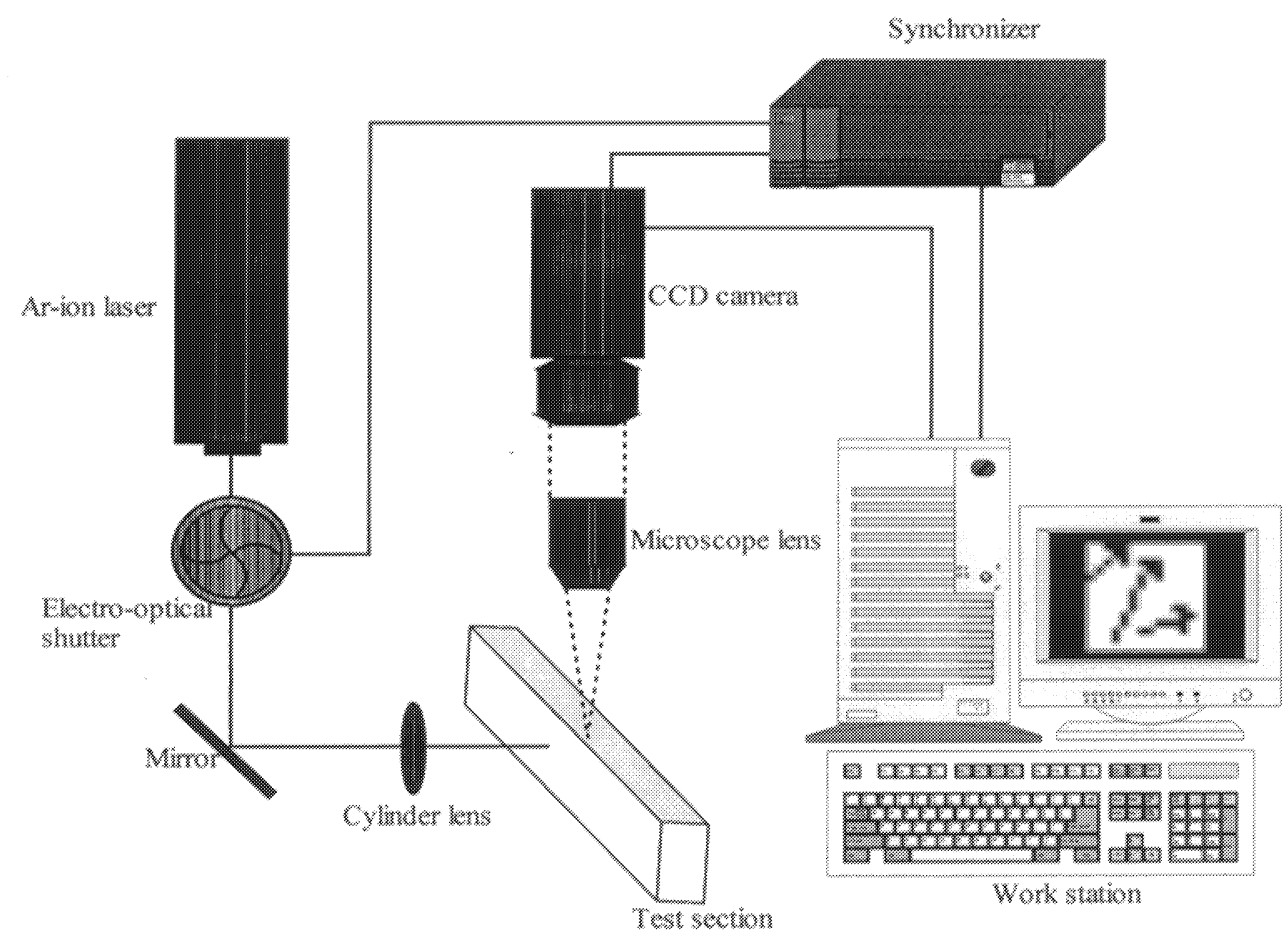

Fig. 2. Schematic of Optical diagnostic setup

\subsection{Data acquisition}

During the given test, the pump and the by-pass valve were adjusted to produce a constant water flow rate. A broad range of flow rates was examined, from $0.65 \mathrm{KPa}$ to over $16.58 \mathrm{KPa}$. These flow rates correspond to Reynolds numbers from 311.3 to 3105.7 . The Re is based on:

$$
\begin{gathered}
Q=\dot{m} / \rho \\
\bar{U}=Q / A \\
\operatorname{Re}=\rho \bar{U} D_{h} / \mu
\end{gathered}
$$

where $\dot{m}$ is mass flow rate, $\mathrm{D}_{\mathrm{h}}$ is hydraulic diameter. Due to velocity of the flow reaches to a few meters per second within millimeter scale at high Reynolds number, exposure time of the CCD should be reduced to microseconds for PIV measurement. In this case, the energy density of the laser is insufficient. So PIV experiment only conducted in low Reynolds number. For high Reynolds number, the flow patterns were recorded use the same optical layout. The configurations of timing sequence were fixed, so that the images of different flow rate can be compared. The length of the particles trace implies the flow velocity. 


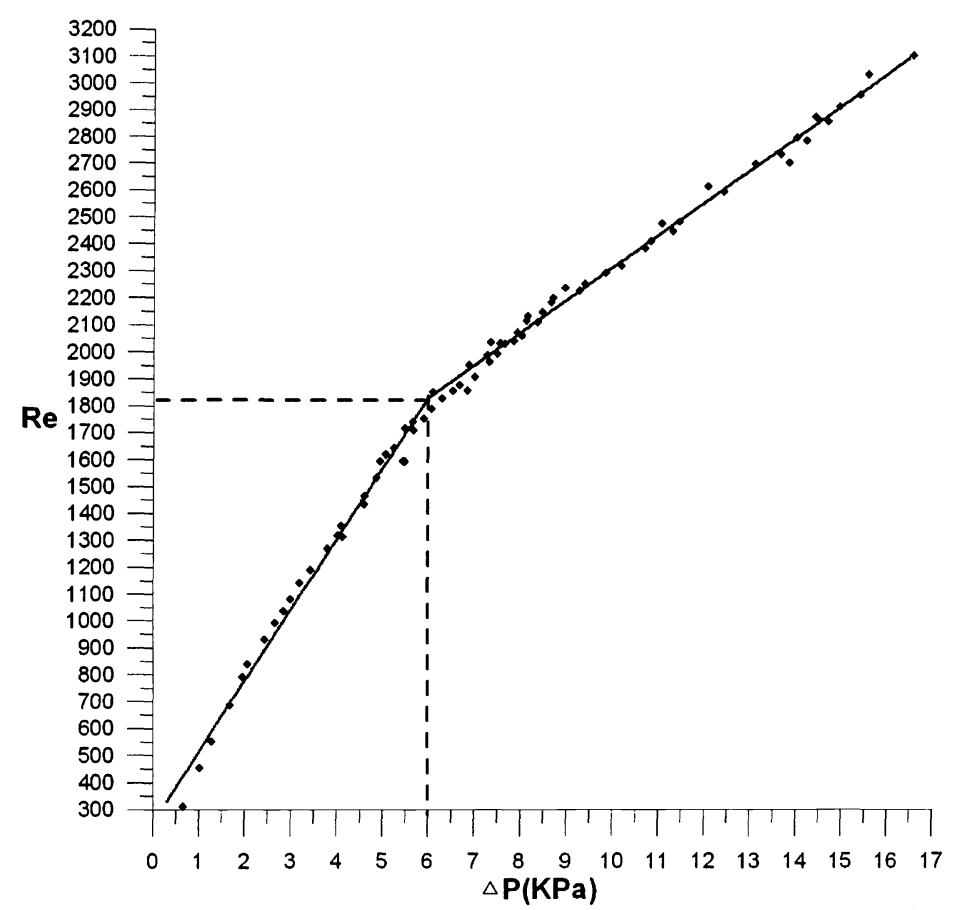

Fig. 3. Pressure drop versus Reynolds number

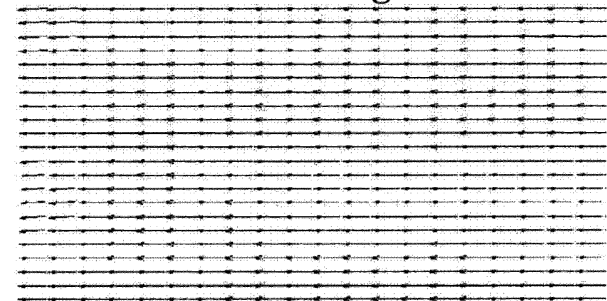

a $\triangle \mathrm{P}=5 \mathrm{KPa}$

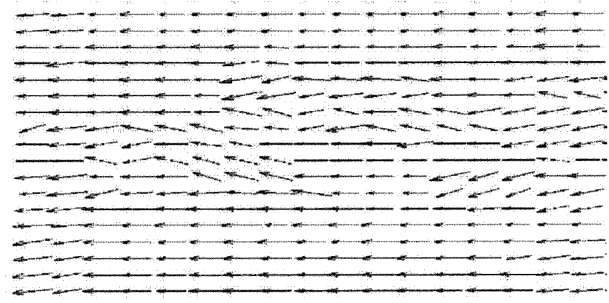

c $\triangle \mathrm{P}=10 \mathrm{KPa}$

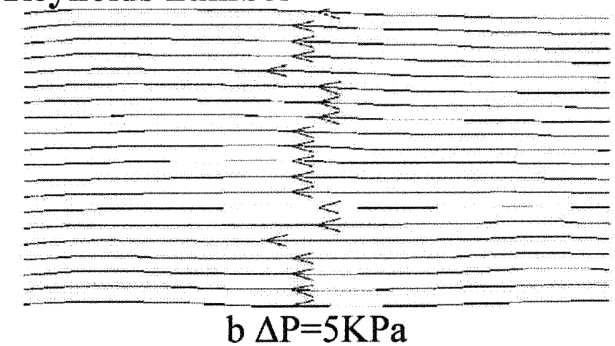

$\mathrm{b} \triangle \mathrm{P}=5 \mathrm{KPa}$

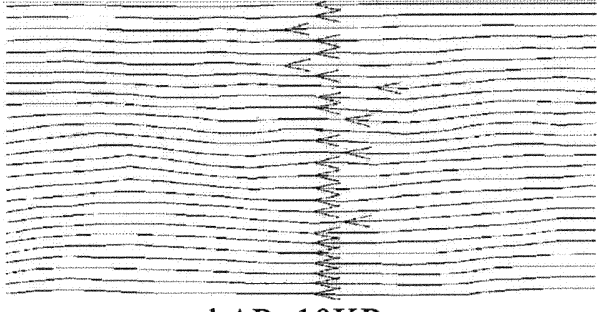

$\mathrm{d} \Delta \mathrm{P}=10 \mathrm{KPa}$

Fig. 4. Vector fields and streamline of the channel flow 


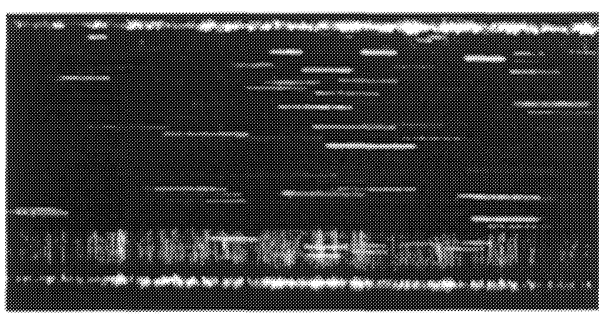

a

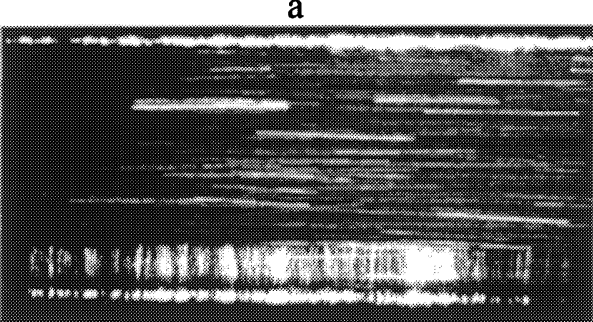

C

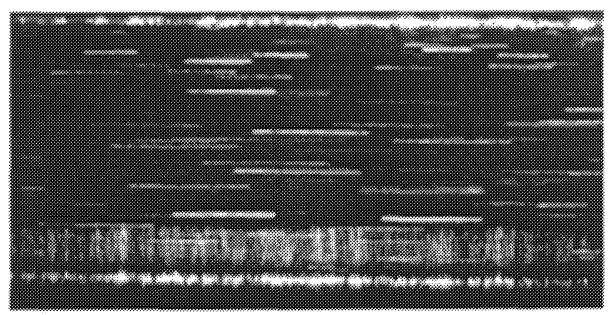

b

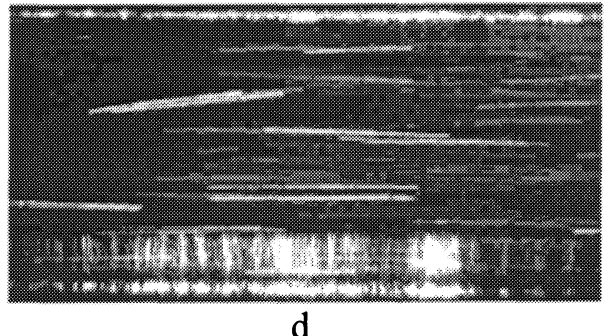

d

Fig. 5. Time sequence of flow visualization

\section{Results and discussion}

Fig. 3 shows the relation of pressure drop versus Reynolds number. The velocity vector fields and diagrams of corresponding streamline are presented in Fig. 4. The vector and streamlines of laminar flow are parallel to the wall. After transition to turbulence, the streamlines were not straight as they were in laminar flow. The direction of the vectors was irregular. It is agreed with the flow visualization results. The images of particle trace shows in Fig. 5. The direction of trace will be random, when turbulence occurred. The transition to turbulence happened when Reynolds number was near 1800 .

\section{Conclusions}

An experimental set-up designed for pressure driven liquid flow through mesoscale rectangular tube has been tested. Transition from laminar flow to turbulent flow occurred in $\operatorname{Re} \sim 1810$. The experimental results show good agreement with predictions of conventional macroscopic theory. Assumptions of no slip and continuum were still valid for explanations of mesoscale flow in these certain conditions. The early transition from laminar to turbulent flow reported in microchannel was never observed in this experiment. Future work will focus on the influence of the slip effect and continuum effect in micron-scale flow. 


\section{References}

[1] Gad-el-Hak M, The fluid mechanics of microdevices-The Freeman Scholar Lecture, J Fluid Eng, 121 pp. 5-33, 1999.

[2] Tuckerman, D. B. and Pease, R. F. W., High-performance heat sinking for VLSI, IEEE Electron Dev. Lett., Vol. EDL-2, No. 5, pp. 126-129, 1981.

[3] Ho C M and Tai Y C, Micro-electro-mechanical systems (MEMS) and fluid flows, Ann. Rev. Fluid Mech. 30, pp. $579-612,1998$

[4] Mala GM, Li D, Werner C, Jacobasch HJ, Ning YB, Flow characteristics of water through a microchannel between two parallel plates with electrokinetic effects. Int J Heat Fluid Flow 18, pp. 489-496, 1997

[5] G. M. Mala, D. Li, J. D. Dale, Heat transfer and fluid flow in microchannels, $A S M E$, 59, pp. 127, 1996.

[6] Mala GM, Li D, Flow characteristic of water in microtubes. Int J Heat Fluid Flow 20, pp. 142-148, 1999

[7] Peng, X. F., Peterson, G. P., and Wang, B. X., Heat Transfer Characteristics of Water Flowing Through Microchannel, Experimental Heat Transfer, Vol. 7, pp.265-283, 1994.

[8] Choi, S. B., Barron, R. F., and Warrington, R. O., Fluid Flow and Heat Transfer in Microtubes, ASME Proceedings, Vol. 32, pp.123-134, 1991.

[9] Harley, J., Huang, Y., Bau, H. \& Zemel, J. Gas flow in micro-channels. J. Fluid Mech. 284, pp. 257-274,1995.

[10] Arkilic, E. B., Schmidt, M. A. and Breuer, K. S., Gaseous Slip Flow in Long Microchannels, $J$. Microelectro- mechanical Systems, 6(2), pp. 167-178, 1997.

[11] Weilin Qu, Issam Mudawar, Experimental and numerical study of pressure drop and heat transfer in a single phase microchannel heat sink, Int J Heat and Mass Transfer, 45, pp. 2549-2565, 2002

[12] SS Mehendale, AM Jacobi, RK Shah, Fluid flow and heat transfer at micro- and meso-scales with application to heat exchanger design, App. Mech. Rev, Vol. 53, No. 7, pp. 175-193, 2000

[13] Qu W, Mala GM, Li D, Pressure driven water flows in trapezoidal silicon microchannels, Int $J$ Heat and Mass Transfer, 43, pp. 353-364, 2000

[14] Carl D. Meinhart and Hongsheng Zhang, The Flow Structure Inside a Microfabricated Inkjet Printhead, $J$. Microelectro- mechanical Systems, Vol. 9, No. 1, 2000.

[15] Xu J, Experimental study on gas liquid two phase flow regimes in rectangular channels with mini gaps, Int $J$ Heat Fluid Flow 20, pp. 422-428, 1999

[16] Meinhart, C. D., Wereley, S.T \& Santiago, J.G., PIV Measurements of Microchannel Flow, Exp. in Fluids, 27 pp. 414-419, 1999.

[17] J. I. Molho, A. E. Herr, T. W. Kenny, M. G. Mungal, P.M. St. John, M. G. Garguilo, D. J. Rakestraw, P. H. Paul, M. Deshpande, and J. R. Gilbert, Fluid transport mechanisms in microfluidic devices, ASME 1998.

[18] Santiago JG, Wereley ST, Meihart CD, Beebe DJ, Adrian RJ, A particle image velocimetry system microfluidics, Exp. in Fluids 25, pp. 316-319, 1998.

[19] Z. Chen, T. E. Milner, D. Dave, and J. S. Nelson, Optical Doppler to-mographic imaging of fluid flow velocity in highly scattering media, Opt. Lett., vol. 22, pp. 64-66, 1997. 